FORMATION Formation emploi

Revue française de sciences sociales

123 | Juillet-Septembre 2013

Pêle-mêle

\title{
Formation et territoires : les contraintes d'une construction partenariale
}

Training and territories: the hardships of building partnership

Berufsausbildung und Gebiete: die Zwänge einer Partnerschaftsaufbau

Formación y territorios : las restricciones de una construcción en conjunto

Jules Simha

\section{(2) OpenEdition}

Journals

Édition électronique

URL : http://journals.openedition.org/formationemploi/4060

DOI : 10.4000/formationemploi.4060

ISSN : 2107-0946

Éditeur

La Documentation française

Édition imprimée

Date de publication : 30 septembre 2013

Pagination : 89-107

ISSN : 0759-6340

Référence électronique

Jules Simha, «Formation et territoires : les contraintes d'une construction partenariale », Formation emploi [En ligne], 123 | Juillet-Septembre 2013, mis en ligne le 08 octobre 2015, consulté le 30 octobre 2020. URL : http://journals.openedition.org/formationemploi/4060 ; DOI : https://doi.org/10.4000/ formationemploi.4060

(c) Tous droits réservés 


\title{
Formation et territoires : les contraintes d'une construction partenariale
}

\begin{abstract}
JULES SIMHA Doctorant en sociologie à I'IDHE (Institutions et Dynamiques Historiques de l'Economie) (UMR nité mixte de recherche 8533), et associé au Centre d'Etudes de I'Emploi (CEE)
\end{abstract}

Résumé

Formation et territoires : les contraintes d'une construction partenariale

Cet article interroge les logiques à l'œuvre dans l'exercice de territorialisation des politiques publiques. S'appuyant sur l'évaluation d'un dispositif régional de territorialisation de la formation professionnelle, il s'agit d'analyser les ressorts d'une telle entreprise, que ce soit en termes de rapport au territoire, ou de relations entre les divers acteurs associés, pour en faire finalement ressortir la dimension de bricolage institutionnel.

Mots clés : politique publique, politique régionale de la formation professionnelle, approche locale

Abstract

Training and territories: the hardships of building partnership

This paper seeks to analyze the ongoing process of "territorialization" of French public policies which tend to give local actors more significance in implementing public policies. More precisely, the research highlights the main characteristics of this process and looks at the ways partners consider the concept of territories, and the relations existing between these partners. Finally, it concludes on the institutional patchwork dimension that seems to best characterize this kind of public policy.

Keywords: public policy, regional CVT policy, local approach

Journal of Economic Literature: $\mathbf{R} 58$

Traduction : Auteur 
La décentralisation entamée au début des années 1980, et poursuivie à travers plusieurs réformes, est intervenue sur un vaste ensemble de prérogatives. Ainsi, la formation professionnelle a été déléguée aux Régions, alors que l'emploi est resté, pour une large part, de la compétence de l'Etat. La difficulté qui résulte de cette séparation institutionnelle n'a pas manqué d'être relevée par exemple par Mossé et Verdier (2003). Quoi qu'il en soit, cette dynamique se poursuit dans un mouvement de territorialisation des politiques publiques. Celui-ci a contribué à instituer le territoire en tant que nouvelle catégorie d'action publique mobilisable par un ensemble d'acteurs relevant aussi bien de la sphère publique que privée (Douillet, 2003).

La territorialisation correspond néanmoins à deux dynamiques distinctes qui renvoient chacune à une conception propre du territoire. Cette distinction se retrouve dans la littérature qui évoque les «logiques de projection» et les «logiques de projet» (Jobert, Guarriello, \& Heidling, 2009), ou les "politiques territorialisées " et les "politiques territoriales" (Hassenteufel, 2008). Dans les deux cas, l'opposition semble se cristalliser autour d'une démarche verticale qui est soit descendante soit ascendante.

La première (la politique territorialisée) se caractérise par une conception par le haut : la collectivité territoriale compétente définit la politique qui s’applique uniformément sur l'ensemble de ses territoires - régions, départements, communes ${ }^{1}$, ou encore bassins d'emploi, zones emploi-formation, etc.

En revanche, pour la seconde (la politique territoriale), la politique émerge directement à son niveau d'application - le territoire - et se conçoit sur la base d'un projet spécifique partagé par l'ensemble des acteurs concernés. Ici, des programmes territoriaux de formation professionnelle se sont développés qui présentent des formes de coopérations multiples et associent acteurs locaux ${ }^{2}$ et collectivités territoriales ${ }^{3}$. Ils cherchent, la plupart du temps, à apparier « besoins économiques » et " demande sociale » en privilégiant une démarche ascendante.

Les deux logiques présentées - descendante et ascendante - doivent être abordées en tant qu'idéaux-types. Evidemment, la réalité des politiques publiques présente un tableau bien moins schématique, et la frontière qui les distingue est loin d'être aussi nette. Toutefois, la question du passage de l'une à l'autre a le mérite de rendre intelligibles des processus et jeux d'acteurs difficilement saisissables. Elle a d'ailleurs déjà été étudiée par Michel Catlla (2004) sous l'angle des relations professionnelles.

1 Par la suite, nous adoptons la convention graphique suivante : Région, Département, et Commune, renvoient aux collectivités territoriales administratives, et lrégion, département, et commune, à leurs zones d'intervention au niveau géographique.

2 Il peut s'agir de maisons de l'emploi, d'agences Pôle-Emploi, de missions locales, d'organismes de formation, etc.

3 Régions, Départements, Intercommunalités et Communes. 
Nous proposons ici un regard différent, celui de la construction d'une politique publique. En effet, dans le cadre de l'observation et du suivi de la conception d'un Programme de Formation Territorialisé, nous cherchons à analyser la posture d'une Région métropolitaine ${ }^{4}$, qu'il s'agisse de ses réalités internes ou de ses interactions avec son environnement. Lanalyse peut être éclairante dans la mesure où elle traite d'une première tentative régionale de territorialisation - au sens " ascendante " - de la formation professionnelle par l'intermédiaire d'un dispositif consacré : auparavant, cette Région ne gérait sa politique de formation professionnelle que de manière descendante.

Dans notre cas, la politique territoriale est impulsée par la Région, et non par les acteurs locaux ; dès lors, il est possible de s'interroger sur son éventuel caractère paradoxal : une démarche ascendante peut-elle être initiée par le haut ? En adoptant une approche sociologique de l'action publique, et en l'articulant à ses dimensions organisationnelles, nous verrons que la politique étudiée relève d'un équilibre à trouver entre bricolage et cadrage institutionnels intervenant à de multiples niveaux.

\section{Encadré 1}

\section{Méthodologique}

L'analyse s'inscrit dans le cadre de l'évaluation triennale d'une « expérimentation sociale » qui a donné lieu à l'observation et au suivi de la construction d'un dispositif de territorialisation de la formation professionnelle, le programme de formation territorialisé (PFT). Ce programme, mis en œuvre par une Région métropolitaine, est destiné aux demandeurs d'emploi de faibles qualifications, et vise leur réinsertion dans l'emploi. L'évaluation a pris la forme d'une observation participante réalisée au sein du Conseil régional, à l'initiative du projet, et de quatre Intercommunalités.

Dans ce cadre, 31 entretiens semi-directifs ont été réalisés avec les différents acteurs impliqués dans le dispositif au niveau régional (directeurs de la formation professionnelle ainsi que du développement économique et de l'emploi, acteur en charge de la conception du programme régional de formation professionnelle, ensemble des chargés de mission), et avec les partenaires de la Région (acteurs de l'emploi, de l'insertion, de la formation professionnelle et du développement économique au sein des Départements, Intercommunalités, mais aussi au sein de Pôle Emploi ou de plateformes de services aux demandeurs d'emploi). Ils portaient sur les différentes représentations qu'ont les acteurs des problématiques d'emploi, de formation professionnelle et de développement économique. Nous nous sommes également intéressés aux dispositifs publics qu'ils mettent en œuvre pour remédier à ces difficultés. Etaient également abordés les obstacles qu'ils rencontraient dans leurs missions.

Ces entretiens et réunions ont tout d'abord été retranscrits, puis analysés de façon thématique. En outre, des séances de restitution ont été organisées auprès des différents acteurs afin d'affiner les hypothèses de recherche.

Nous nous appuyons en outre sur une analyse documentaire des rapports internes au Conseil régional ainsi que sur l'observation en direct de 36 réunions et comités de pilotage auxquels nous avons participé et qui associaient l'ensemble des acteurs intéressés au projet.

4 Les noms et acronymes des dispositifs et des collectivités territoriales ont été anonymisés. 
La notion même de territoire - au cœur de la logique de territorialisation - est une construction dont la Région peut difficilement faire l'économie, et ce d'autant plus lorsqu'elle ne dispose pas de relais territoriaux institués (partie 1). Les conflits propres au Conseil régional illustrent alors ses tâtonnements et ses tentatives d'appropriation et de (re)définition du territoire (partie 2). Mais la singularité de ce type de démarche relève de l'effort de co-construction réalisé par un ensemble de partenaires, notamment constitué de collectivités territoriales, autour d'un " projet commun » (partie 3).

\section{Le « territoire pertinent »: résultat d'une logique hybride}

Dans une Région où la conception de la formation professionnelle répond à une démarche descendante, le programme de formation territorialisé (PFT) a été présenté comme l'opportunité d'une nouvelle procédure de commande et de gestion de la formation. Dans cette perspective, les acteurs en charge au niveau régional ont cherché à établir un cadre au sein duquel la notion de " territoire pertinent " joue un rôle central.

Nombreux ont été les travaux qui se sont penchés sur la question du territoire, l'abordant sous divers regards et s'efforçant de rendre compte de ses multiples facettes. (Jobert, Guarriello, \& Heidling, 2009 ; Rey, 2012). Pour autant, le sujet est loin d'être épuisé. En témoignent, notamment, les différentes acceptions que lui prêtent le technicien et l'expert. En effet, une frontière demeure visible lorsque l'on s'attache à la manière dont le premier se saisit des concepts élaborés par le second. Le cas dont il est question ici permet d'illustrer cette distinction entre technicien et expert. Plus spécifiquement, nous soulignons une première difficulté rencontrée par le Conseil régional. Cette difficulté relève aussi bien de la manière dont celui-ci cherche à définir un " territoire pertinent ", que de son positionnement entre logique ascendante ou descendante s'agissant de la conception de sa politique de formation professionnelle.

\subsection{Définir un « territoire pertinent »}

Alors que dans la plupart des Régions, des instances et des référents territoriaux ont été institués en matière de formation professionnelle (Lamanthe, 2004), celle que nous avons étudiée se caractérise par une absence de tels relais territoriaux. Or, ces derniers participent largement d'une définition du territoire qui peut correspondre à leurs zones d'intervention. Ainsi, notre Région était relativement dépourvue de supports sur lesquels la définition du « territoire pertinent " aurait pu prendre corps. Dans ce contexte, son "Service PFT " a cherché d'autres éléments de cadrage lui permettant de délimiter le territoire pertinent par rapport à son objectif de territorialisation. 
A cette occasion, il est possible de retrouver différentes acceptions du territoire évoquées ci-dessus. En effet, d'un côté, le directeur du service chargé du PFT met en avant l'idée de " territoire de projet »:

"On a du mal à déterminer ce quion appelle un territoire d'intervention, et on a fini par dire que le bon territoire cohérent, c'est le territoire qui correspond au projet. » (Directeur du service PFT, 6 ans passés à la Région, anciennement directeur adjoint d'une structure para-régionale de formation professionnelle)

De l'autre, il désigne la Communauté d'agglomération comme structure porteuse du PFT, et donc comme périmètre d'intervention. Enfin, il définit le territoire pertinent en fonction d'un critère de temps : une cartographie des organismes de formation situés à 45 minutes des Communautés d'agglomération.

Territoire de projet, périmètre administratif, et zones de mobilité sont ainsi sollicités dans cet effort de définition. L'utilisation par le Conseil régional de trois conceptions différentes, voire opposées, du territoire souligne le caractère malléable de ce dernier. Ainsi, le territoire n’est pas un "donné " a priori, mais un "construit ", fonction des réalités auxquelles les acteurs doivent faire face.

Toutefois, si le concept de " territoire de projet " renvoie bien à une logique ascendante (Jobert, 2009) en ce sens qu'il est le fruit d'un partenariat local, la manière dont les deux autres critères sont déterminés relèverait plutôt d'une démarche descendante : la Région définit elle-même le territoire à partir duquel le projet devra être élaboré.

\subsection{La Région entre tradition et innovation}

Les nombreux travaux, comme ceux de Muller (2005) ou Lascoumes \& Le Galès (2004) ayant abordé la question du changement en politique publique s'accordent sur la relative lenteur de ce processus. Si nous ne pouvons, ici, parler d'un changement de politique publique dans la mesure où l'analyse intervient sur le temps court, il n'en reste pas moins que pour les élus et les services régionaux, le PFT revêt un caractère innovant. Un rapport du Conseil régional évoque ainsi « linversion de logique » dont il est porteur. Cette inversion ne répond pourtant pas à une démarche idéale-typique. Pour comprendre la posture intermédiaire adoptée par le service en charge du PFT, il est intéressant de focaliser le regard sur ses habitudes, confrontées à ses aspirations en termes de territorialisation.

Pour le service PFT, le choix de la Communauté d'agglomération se justifie par la volonté régionale de produire une politique de territorialisation qui intègre les problématiques de formation professionnelle à celles relatives à l'emploi et au développement économique. Or, la territorialisation de ces deux domaines était déjà engagée et se concrétisait par une contractualisation entre le Conseil régional et les Communautés d'aggloméra- 
tion $^{5}$, collectivités notamment compétentes en matière de développement économique ( $c f$. encadré 2 ). Il a ainsi été décidé que le PFT serait porté au niveau infra-régional par les Communautés d'agglomération.

Cet exemple illustre la délicate posture dans laquelle se trouve la Région qui, pour reprendre la notion de Pierre Muller (2005), cherche à articuler deux "référentiels " : celui d'une logique de projet, et celui d'une logique de projection (Jobert, Guarriello, \& Heidling, 2009). La difficulté se retrouve d'ailleurs dans le discours des acteurs du service chargé du PFT, qui fait apparaître deux postures, relevant chacune d'un des deux référentiels.

La première met en avant le Conseil régional en tant que " chef de projet »:

"On se vivait comme détenteurs, concepteurs et maitres d'œuvre de la politique publique globale "(Directeur de la direction au développement économique et à l'emploi, 38 ans, six ans passés auparavant à divers postes du Conseil régional)

Alors que la seconde relève plutôt d'une mise en retrait de la Région par rapport aux autres partenaires, quitte à laisser à qui veut cette place de premier plan :

"Si on [réussit] à faire en sorte que les territoires arrivent à faire l'animation des acteurs locaux, la Région apportant simplement ses ressources pour financer la formation, [et que] le Conseil général, Pôle emploi, les missions locales ou autres acteurs apportent leurs ressources pour accompagner, etc., on aura quand même fait un grand pas. " (Chargée de mission PFT, 38 ans, six ans passés à la Région. Anciennement chargée d'études sur les questions d'éducation au sein d'une Commune)

L'ouverture du périmètre de la Communauté d'agglomération à une zone de mobilité à 45 minutes reflète une démarche identique. Dans l'esprit du service chargé du PFT, « les 45 minutes" sont l'occasion de proposer un territoire qui corresponde aux problématiques de formation professionnelle.

Mais cette démarche de construction a été déterminée et élaborée de manière unilatérale, c'est-à-dire sans consulter les acteurs locaux... pourtant au fait des habitudes et des besoins de leur population. En effet, pour les acteurs du Service PFT, ces derniers ne sont pas compétents sur ce point :

"Mais cette méthodologie d'analyse de l'offre de formation, c'est quelque chose qu'on a pris en charge parce qu'effectivement, on constatait que les territoires ne pouvaient pas, ne savaient pas le faire. " (Directeur du Service PFT)

Une fois de plus, il est intéressant de constater un décalage entre la notion de territoire de projet " qui est défini par la nature et les objectifs des projets, les acteurs qui les portent et l'action collective qu'ils déploient pour le faire aboutir " (Jobert, 2006), et la manière dont cette construction a été réalisée sans le concours de ces acteurs locaux. En outre, le ter-

5 Ce point est développé en deuxième partie. 
ritoire des 45 minutes n'a pas convaincu ces acteurs; ils estimaient parfois qu'il n'avait aucune légitimité puisque certaines communes n'avaient accès, par l'intermédiaire de ce critère, à aucun organisme de formation.

Il semble donc que le Conseil régional se trouve tiraillé entre sa volonté " d'inverser la logique ", ce qui passe par l'association de nombreux acteurs, par leur mise en réseau et la constitution de concertations territoriales ${ }^{6}$, en somme par l'élaboration d'une forme de gouvernance territoriale, et l'ancien référentiel producteur d'une résistance à l'innovation.

La question de la construction du « territoire pertinent ", que l'on peut considérer comme l'une des premières étapes de l'élaboration du PFT, permet ainsi d'avancer que la politique de territorialisation produite par le Conseil régional ne répond finalement ni totalement à la logique ascendante, ni totalement à la logique descendante, mais plutôt à une logique hybride. Il est en effet indéniable que le PFT introduit une nouvelle démarche de conception de la formation professionnelle pour le Conseil régional. Celui-ci a d'ailleurs été présenté comme un dispositif de co-construction dans la mesure où il accorde une place importante aux acteurs locaux dans l'identification et l'analyse des besoins. Cependant, la difficulté avec laquelle le Conseil régional, et plus précisément son Service PFT, manie cette nouvelle dimension territoriale, pondère ce premier constat.

Afin de comprendre ce paradoxe apparent, il peut être intéressant de s'intéresser aux tractations à l'origine de l'introduction de l'objectif de territorialisation au Conseil régional.

\section{L'opposition des stratégies d'acteurs au sein du Conseil régional}

Le Conseil régional est une organisation complexe qui rassemble une large palette d'acteurs regroupés au sein d'unités, de directions, sous-directions et services dont la structure hiérarchique n'exclut pas les points de vue divergents, voire les oppositions. Cette présentation succincte rappelle tout à fait l'un des éléments de caractérisation de l'organisation par les tenants de la sociologique des organisations : elle est « un lieu d'affrontement constant entre des intérêts plus ou moins divergents sinon contradictoires où il importe de saisir comment la situation de travail offre à certains le moyen d'obliger les autres à plier devant eux" (Friedberg, 1988). Ces travaux proposent une analyse des relations de pouvoirs au sein des organisations ainsi que dans leur rapport à l'environnement. Dans notre cas, ils permettent une analyse des négociations qui ont eu lieu au moment de la création du PFT, sans pour autant caricaturer les jeux d'acteurs prenant part au processus.

6 En témoigne notamment un rapport du Conseil régional de 2008. 
Ainsi deux logiques principales semblent dialoguer au sein du Conseil régional ; elles relèvent des relations et points de vue de deux catégories d'acteurs, les élus et les techniciens, qui ont scandé le rapport de la région à ses territoires. Ainsi, en contournant les résistances des techniciens, les élus ont contribué à la territorialisation de la formation professionnelle.

\subsection{Elus et techniciens, deux visions du territoire}

Au sein du Conseil régional, la question territoriale a mobilisé deux principaux groupes d'agents. Les premiers sont les élus régionaux. Ce sont eux qui fixent les stratégies et les fils rouges de la politique régionale. Les deuxièmes sont les techniciens qui conçoivent et appliquent les dispositifs, concrétisation des politiques décidées par les élus.

Pour simplifier la compréhension, on peut distinguer deux temps qui caractérisent la vision de ces deux groupes sur la territorialisation, et, partant, leurs relations mutuelles.

- Tout d'abord, élus et techniciens partageaient une même position relative à l'engagement du Conseil régional sur ses territoires. A l'époque, la territorialisation était traditionnellement évitée conformément au discours officiel selon lequel « il n’y a pas de territoires » dans cette région. Par conséquent, la politique y était conçue par le haut sans donner de crédit au discours en voie de généralisation favorable aux rapports de proximité (Lamanthe, 2004). D'une certaine manière, ce premier temps correspond à la situation d'équilibre du système social de l'organisation dans laquelle les participants sont tous satisfaits (Friedberg, 1988).

- Puis dans un second temps, cette position a commencé à perdre de son caractère officiel (Agulhon, Trautman $\&$ Vincent, 2002). Les élus régionaux en sont venus à réclamer l'élaboration d'une réponse spécifique aux besoins locaux de formation professionnelle. Mais leurs demandes et pressions se sont heurtées à la résistance des techniciens qui, eux, n’avaient pas renouvelé leur position et qui défendaient une vision totalement unifiée du territoire :

"La formation professionnelle, ça couvre un bassin d'emploi, et [ici], le bassin d'emploi, c'est la Région» (Directeur de la direction au développement économique et à l'emploi).

En dépit de la faible marge de manœuvre dont disposent les techniciens vis-à-vis des élus, ils ont néanmoins réussi, en tout cas pour un temps, à instaurer une situation de statu quo. Elle peut se comprendre si l'on considère un petit dispositif à visée adéquationniste dont disposaient les techniciens de la formation professionnelle. Par l'intermédiaire d'une

7 L'approche adéquationniste renvoie à une vision de la relation formation-emploi figée dans l'instant. Elle correspond à " une vision traditionnelle des relations entre formation et emploi laissant entendre qu'une adéquation fine et individuelle est possible entre des contenus d'emploi et des contenus de formation " (Rose, 2008). 
entrée " entreprises ", il proposait des actions de formation courtes et très spécifiques, relevant essentiellement de l'adaptation aux besoins ponctuels.

La stratégie des techniciens consistait alors à utiliser ces actions en tant que caution territoriale pour répondre aux demandes des élus :

"C'était longtemps présenté comme une espèce de talisman, en disant "si si! regardez! On a territorialisé!" Alors bon, cétait complètement épiphénoménal, mais peu importe» (Idem)

\subsection{Le pouvoir du « local»}

Au vu de ces premiers éléments, il pourrait être tentant de se représenter ces deux groupes selon un deuxième critère de distinction, la séparation géographique : les élus seraient concentrés au niveau régional alors que les techniciens interviendraient plus localement. En réalité, le jeu est plus complexe dans la mesure où l'un et l'autre interviennent - plus ou moins indirectement - dans la sphère du deuxième.

Tout d'abord, nous avons vu que les techniciens ne sont pas diligentés sur les territoires, et conçoivent l'offre de formation au niveau régional. En outre, les élus d'un Conseil régional disposent très souvent d'un double mandat. Ils sont alors élus locaux avant d'être élus régionaux ${ }^{8}$, et leurs demandes au niveau régional recoupent très souvent les besoins du niveau local. Ils sont donc en mesure de faire remonter à l'échelon régional les dysfonctionnements qu'ils identifient sur leurs territoires.

Aussi, les demandes de territorialisation qui peuvent être analysées comme la volonté de s'inscrire dans de nouvelles démarches faisant la part belle à la démocratie de proximité, sont également compréhensibles au prisme des logiques dites clientélistes. Un de nos enquêtés, directeur adjoint d'un Vice-président du Conseil général, insistait à ce propos sur les demandes locales qui cherchent à se démarquer de celles des territoires voisins afin d'attirer les financements régionaux pour des besoins affichés comme spécifiques. Or, selon lui, bien que la Région considère rarement ces besoins comme spécifiques - parce qu'elle a pu, par ailleurs, les identifier à l'échelle régionale - elle continue de développer des réponses « adaptées » et d'y adjoindre une ligne financière.

Une explication peut être apportée si l'on considère les intérêts en présence. D’une part, les élus sont satisfaits parce qu'ils obtiennent gain de cause et peuvent afficher, sur leur circonscription, leur capacité à attirer une aide spécifique de la Région. D’autre part, les techniciens n'interviennent qu'à la marge puisqu'il ne s'agit pas, dans ce cas, de concevoir un nouveau programme, mais seulement de modifier la forme de l'existant.

8 Au sein du Conseil régional, plus de la moitié des sièges sont attribués à des conseillers régionaux qui cumulent un deuxième mandat. 
Dans le même temps, les techniciens dénoncent ces comportements sous le vocable de " logique de guichet » qui constituerait une entrave à la démarche de territorialisation que la Région tenterait de mettre en œuvre par l'intermédiaire du PFT :

"La Région est amenée à jouer un rôle d'animateur etlou de coordonateur, ce qui n'est pas toujours bien compris par les acteurs locaux qui la considèrent souvent comme un tiroir-caisse, dans la mesure où pour eux [les acteurs locaux], il est surtout question de bénéficier de l'apport pécuniaire régional. " (Directeur du Service PFT)

La question de la territorialisation de la formation professionnelle mobilise ainsi deux groupes principaux aux intérêts et ressources très différents. En définitive, elle n’a été rendue possible que par l'intermédiaire d'une stratégie de contournement de la part des élus : devant l'opposition des techniciens, les élus ont utilisé le mouvement de territorialisation en matière de développement économique et d'emploi comme un catalyseur.

\subsection{Contourner les résistances}

Ce mouvement de territorialisation a été initié au Conseil régional suite à des concertations territoriales organisées en vue d'élaborer le Schéma Régional de Développement Economique ${ }^{9}$. Un des points d'achoppement relevés à cette occasion a été celui d'une insuffisante territorialisation de l'action économique régionale :

"Vous [la Région] êtes insuffisamment territorialisés, on ne vous connaît pas, on ne sait pas ce que vous faites. " (directeur de la Direction au développement économique et à l'emploi).

En réaction, la Direction régionale au développement économique et à l'emploi a élaboré un nouveau dispositif, le "Pacte ", dont l'objectif premier serait la territorialisation du SRDE. Les élus régionaux qui, on l'a vu, insistaient depuis plusieurs années pour mettre en œuvre la territorialisation de la formation professionnelle se sont appuyés sur le « Pacte " pour exiger cette dernière.

Ils ont ainsi élaboré une stratégie visant à éviter la contrainte posée par les techniciens. Arguant de la nécessité d'articuler les trois dimensions, au lieu de les concevoir de manière segmentée, ils ont imposé la greffe de la formation professionnelle aux côtés de l'emploi et du développement économique comme domaine d'intervention du « Pacte ». Cela s'est traduit par l'intégration du PFT au Pacte selon la logique des structures emboîtées évoquées habituellement par l'image des "poupées russes ».

Ce rapide retour sur les stratégies des acteurs régionaux visant à éviter ou à imposer un nouveau dispositif fournit un bon aperçu de la manière dont chacun cherche, à sa manière, à préserver ses propres intérêts. Il permet également de mieux appréhender les

9 Le SRDE définit les orientations de la Région en matière de développement économique. 
difficultés rencontrées par les techniciens pour élaborer un cadre territorial pertinent. Il illustre enfin la réalité de la construction de ce dispositif qui, par de nombreux aspects, semble plus relever du bricolage institutionnel que d'une construction rationnelle et stratégique.

Pour compléter ce tableau, il est intéressant d'examiner les rapports que le Conseil régional entretient avec son environnement dans le cadre de la mise en œuvre de sa politique de territorialisation de la formation professionnelle. Le PFT a, en effet, été conçu comme un outil de dialogue avec les partenaires impliqués.

\section{Une construction partenariale 3 de la territorialisation}

L'inversion de logique évoquée dans les rapports du Conseil régional à propos du PFT relève tout autant de la nouvelle entrée territoriale que ce dernier est appelé à investir que de l'ouverture à un partenariat élargi. D'ailleurs, pour certains techniciens, son principal intérêt ne résiderait pas tant dans sa dimension territoriale que dans sa dimension partenariale. Dès lors, l'approche peut difficilement se soustraire à un regard sur l'environnement du Conseil régional constitué de la multitude des agents qui interviennent à différents niveaux. En raison de cette grande diversité, les développements suivants restreignent l'analyse aux collectivités territoriales ${ }^{10}$.

Cette dernière partie s'interroge spécifiquement sur le choix du Conseil régional d'associer, dans sa démarche de territorialisation de la formation professionnelle, des acteurs qui ne sont légalement pas compétents en la matière, comme l'illustrent les attributions dévolues aux collectivités territoriales associées au projet (encadré 2). Elle montre que si l'on déplace l'angle d'approche - du Conseil régional vers ces acteurs, mais aussi de la question de la formation professionnelle à celle de la territorialisation leur association prend un autre visage. On envisagera successivement ici la place des Départements, des Communautés d'agglomération puis des Régions.

10 Pour une analyse des relations Région-Etat et formation professionnelle-emploi, voir notamment Bel \& Dubouchet, (2004), ou Berthet (2010). 


\section{Encadré 2}

\section{La formation, l'insertion, l'emploi et le développement économique :

$$
\text { quelle répartition? }
$$

Au terme des lois successives de décentralisation, l'Etat a transféré certaines de ses compétences aux collectivités territoriales. Très schématiquement, les responsabilités en matière d'emploi, de développement économique, d'insertion et de formation professionnelles peuvent se définir de la manière suivante :

En matière de formation professionnelle, la Région est responsable des demandeurs d'emploi jeunes et adultes. A ce titre, elle est chargée d'élaborer un Plan Régional de Développement des Formations Professionnelles (PRDFP) qui présente sa stratégie à moyen terme. Depuis la loi relative à l'orientation et à la formation tout au long de la vie du 24 novembre 2009, ce document a intégré une dimension contractuelle (on parle depuis du CPRDFP), et lie le Conseil régional et I'Etat (et le Recteur). Pour cette raison, le CPRDFP a été fortement décrié par plusieurs Régions qui ont pu y voir une tentative de « recentralisation » des politiques de formation professionnelle $\left(^{*}\right)$.

Le Département et la Commune ne sont pas compétents en ce domaine.

En matière de développement économique, la compétence a également été décentralisée à la Région. Cette dernière définit un Schéma Régional de Développement Economique (SRDE) et coordonne l'action des autres collectivités sur ce plan $\left.{ }^{* *}\right)$. Départements et Communes peuvent ainsi participer à la politique de développement économique portée par la Région.

En ce qui concerne l'insertion professionnelle (et sociale), et plus précisément le RSA (revenu de solidarité active), le Département est responsable. Il peut néanmoins en déléguer la charge à des organismes spécifiques.

Enfin, la compétence en matière d'emploi relève toujours de l'Etat qui l'exerce grâce au concours de ses services déconcentrés (Direction régionale des entreprises, de la C Concurrence, de la consommation, du travail et de l'emploi-Direccte, Préfecture de région, etc.). Toutefois, missions locales et permanences d'accueil, d'information et d'orientation (PAIO), par exemple, peuvent être mises en place par les collectivités territoriales, qui participent, en outre, à leurs financements. De même, les maisons de l'emploi qui sont labellisées au niveau national, sont souvent mises en place à l'initiative des collectivités territoriales.

(*) Dépêche AEF n¹38189, Midi-Pyrénées : la négociation du CPRDFP sera « un exercice délicat ».

(**) Article L1511-1 du Code général des collectivités territoriales.

\subsection{Les Départements, un affichage politique}

Afin de comprendre les raisons pour lesquelles le Département a été associé au PFT, il est important de considérer, tour à tour, les motivations du Conseil régional - initiateur de la démarche - et celles du Conseil général. En effet, chacun y trouve un moyen de situer son action prioritairement en regard de ses enjeux propres. La question est légitime dans la mesure où la Région décide d'associer à la construction de son dispositif un acteur qui n'est pas légalement compétent en la matière, et qui de surcroît ne semble pas correspondre à sa définition du " territoire pertinent». 
Toutefois, il présente, pour le Conseil régional, un certain nombre d'avantages au premier rang desquels se trouvent les populations pour lesquelles il définit ses politiques. En effet, les bénéficiaires cibles du PFT sont très proches de ceux pris en charge par le Département au nom de sa compétence en insertion professionnelle. Cette affinité de publics rencontre, en outre, la volonté du Conseil régional de décloisonner les logiques de financement en fonction des statuts des individus : le PFT propose des parcours à destination de tous les individus non salariés ${ }^{11}$, jeunes ou adultes, bénéficiaires ou non du RSA (revenu de solidarité active). La logique est alors celle d'un financement conjoint des actions de formation, là où généralement " chacun subventionne ses propres bénéficiaires ", comme le souligne cet acteur local de l'insertion :

"Le Conseil régional, il faudrait quill travaille un peu avec le Conseil général (...) chacun fait son programme [de formation, d'insertion] de son côté." (directrice d'un Plan Local pour l'Insertion et l'Emploi, 50 ans)

Enfin, pour la Région, la participation du Département confère à son nouveau dispositif une forte dimension politique. Elle permet, en effet, non seulement d'afficher la construction de nouveaux partenariats, mais aussi de renforcer les dimensions d'analyse des besoins que le PFT est censé venir combler.

En ce qui concerne le Conseil général, il semble que cette dimension d'affichage politique soit une des principales motivations l'ayant poussé à la contractualisation avec le Conseil régional. En effet, sa participation au PFT lui permet de mettre en avant son soutien à certains territoires. Un acteur du Conseil général associé à la démarche du PFT expliquait ainsi les raisons pour lesquelles le Département avait été associé tardivement à la démarche de territorialisation sur une Communauté d'agglomération :

"Parce quill y a eu des hésitations des élus du [Conseil général], est-ce qu'on y va, est-ce qu'on y va pas... ? Il y a une question de moyens, mais je pense que la vraie raison c'est plutôt les relations entre le [Conseil général] et [ce territoire]. Et, quelques mois ont passé, on a fait une note, et puis finalement, la direction générale [du Conseil général] a dit oui on y va, on ne peut pas se passer du Département. C'est un peu impensable que le Département ne soit pas présent sur des choses comme ça. " (chargée de projet au sein du service du développement économique, de l'emploi et de la formation professionnelle du Conseil général, 45 ans, depuis 2008 au Conseil général. Anciennement au service jeunesse d'une mairie)

En l'occurrence, cette Communauté d'agglomération est considérée par le Conseil général comme étant déjà très structurée, et n’ayant donc pas besoin de soutien supplémentaire. Ce dernier décide néanmoins de s’investir sur ce territoire et donc de ne

11 Les Régions ne sont pas compétentes pour la formation professionnelle des salariés, qui relève des sphères d'intervention des partenaires sociaux et de l'Etat. 
pas faire l'impasse sur ce genre d'initiative, quand bien même il n'apporterait aucun financement.

Parmi les territoires sur lesquels nous avons observé la construction du PFT, l'un d'entre eux - que nous appellerons $\mathrm{AB}$ - nous parait constituer une bonne illustration de la relation qui se joue entre Département et Région.

Sur $\mathrm{AB}$, les acteurs emploi et insertion cherchent à utiliser toutes les ressources à leur disposition afin de pallier l'enclavement structurel du territoire. Au cours des réunions territoriales, la position en retrait de la Région a offert l'opportunité au Département de prendre l'initiative d'un parcours de formation. En réalité, il s'agissait principalement pour ce dernier de valoriser une action existante. De la sorte, il n'aurait pas à consentir une nouvelle ligne budgétaire. Dans la mesure où les acteurs de $\mathrm{AB}$ ne se trouvaient pas en position de refuser, et parce qu'ils pensaient avoir identifié un besoin correspondant, cette action a été mise en œuvre. La Région, initiatrice de la démarche de territorialisation, a finalement été exclue du processus parce que le PFT n'était pas compatible avec l'action du Département dans la mesure où celle-ci ne pouvait intégrer l'idée de parcours de formation, constitutive du PFT.

Cet exemple permet de revenir sur les stratégies des acteurs régionaux.

Les élus, tout d'abord, semblent avoir obtenu gain de cause puisque les techniciens sont intervenus sur le territoire et ont participé à la construction d'une action de formation. Les techniciens, quant à eux, ont répondu aux attentes des élus tout en laissant à un partenaire le soin de produire l'innovation. Le Département, enfin, peut se prévaloir d'avoir joué un rôle de premier plan sans, pour autant, avoir élaboré une nouvelle réponse.

\subsection{Les Communautés d'agglomération, une vision complémentaire}

Du côté des Communautés d'agglomération, la question se pose différemment. Si, à l'exemple des Départements, elles ne sont pas compétentes en matière de formation professionnelle, elles ont néanmoins été identifiées par la Région comme un acteur important de sa politique de territorialisation. En effet, la Communauté d'agglomération apporte une vision complémentaire de l'approche régionale des besoins. Là où la Région raisonne à une échelle globale, la Commune ou l'Intercommunalité raisonne à un niveau bien plus local du fait de sa connaissance fine des publics et entreprises de son territoire. Pourtant, ainsi que le relate ce directeur d'une Communauté d'agglomération, la complémentarité n'est pas toujours acquise :

"On avait (...) des échanges, des oppositions presque fondamentales. A savoir que nous, on disait, il y a des opportunités d'emploi, et bien si on collait à ces opportunités d'emploi ?, et elles nous disaient, ah non non, c'est pas la philosophie de la Région! Quels sont les projets de vos jeunes?, et si on travaillait à partir de vos projets?" (45 ans, depuis 5 ans à son poste). 
L'opposition rapportée par cet acteur renvoie à la difficulté pour le Conseil régional et ses partenaires à articuler les deux référentiels descendant et ascendant. Plus généralement, nous retrouvons ici une opposition structurelle entre les collectivités territoriales, qui renvoie à des conceptions différentes de l'allocation des ressources. Bel (2007) souligne à ce titre que "des conflits d'intérêts, des concurrences peuvent émerger entre une logique d'aménagement du territoire, portée par la Région, visant une répartition équitable des moyens et une logique de développement territorial, portée par des Communes ou conseils généraux, visant le développement du territoire».

En définitive, tout le jeu des concertations territoriales organisées en vue de la construction du PFT tourne donc autour du poids accordé, par chaque acteur, à ces deux angles d'approche. Sur le terrain, deux territoires illustrent la manière dont l'une ou l'autre de ces approches a pu prendre le dessus sur l'autre.

Sur BC, les techniciens régionaux se sont peu investis, et n'y ont pas porté clairement le discours propre à la démarche ascendante. Aussi, les acteurs de la Communauté d'agglomération n’ont jamais intégré cette logique. Pour eux, la territorialisation relève plutôt d'ententes entre élus locaux et régionaux. Par conséquent, le PFT n'a pas été concrétisé ; l'ensemble des acteurs régionaux et locaux continue de travailler selon leurs habitudes.

En revanche, $\mathrm{AC}$ a fait montre d'une réelle capacité à organiser des concertations territoriales et à recueillir les divers points de vue. La mise en retrait du technicien en charge de la formation professionnelle n'a pas empêché les partenariats locaux de se saisir de la question de la territorialisation. Prenant acte de l'absence du technicien, la Communauté d'agglomération, alors en construction, a monté un projet de PFT en toute autonomie.

Dans le rapport à leur environnement, nous retrouvons une stratégie commune des techniciens qui consiste donc à laisser une grande marge de manœuvre aux acteurs locaux. Si ceux-ci s'en saisissent, le dispositif peut émerger. En l'occurrence, une différence importante entre $\mathrm{BC}$ et $\mathrm{AC}$ réside dans l'ancienneté de la Communauté d'agglomération. Créée il y a plusieurs années pour BC, elle était encore en construction pour AC. Aussi, les acteurs locaux fédérés autour de la Communauté d'agglomération n'avaient ni les mêmes habitudes de travail, ni les mêmes intérêts à les modifier dans la perspective de l'élaboration d'un PFT.

\subsection{La Région : quelle coordination territoriale ?}

L’un des objectifs affichés de la Région, lorsqu'elle décide de mener la territorialisation de la formation professionnelle, est d'inverser la logique à l'œuvre dans les sollicitations émanant des acteurs locaux : le principe du " guichet " doit être abandonné, et la Région doit être considérée comme pilote et partenaire des projets territoriaux. Elle se pose alors comme instigateur d'une démarche de territorialisation que les acteurs locaux doivent s'approprier. En quelque sorte, la Région pose les règles du jeu auxquelles devront se soumettre l'ensemble des acteurs désireux d'intégrer cette nouvelle 
logique de régulation. Elle se saisit donc de sa compétence en matière de formation professionnelle pour affirmer son rôle de pivot : elle est à la fois l'acteur par lequel la territorialisation est initiée, et celui qui participe à sa mise en œuvre.

Toutefois, cet affichage politique n'a trouvé qu'une traduction très limitée en termes de moyens et de personnels. En effet, les chargés de mission PFT ne sont pas spécifiquement attachés au PFT, et la direction à la Formation professionnelle du Conseil régional n'est pas organisée pour "aller sur le terrain " : ses personnels ne sont pas qualifiés pour mener à bien une mission de coordination à une échelle territoriale, qui requiert une connaissance fine des acteurs locaux de l'emploi, de la formation, et de l'insertion - ce que la Communauté d'agglomération a pu, par ailleurs, lui apporter.

Relativement dépourvus de solution pour résoudre ces difficultés, les techniciens ont eu tendance à laisser la main de la coordination à d'autres acteurs, et à adopter, au mieux, une position de simples partenaires, comme l'illustrent les exemples des trois territoires évoqués ci-dessus. Les conséquences de ce positionnement délicat - une prise en étau entre une volonté politiquement affirmée (les élus) et l'absence de moyens fournis pour s'en faire l'écho (les techniciens) - sont multiples et dépendent des configurations territoriales préexistantes. Celles-ci ne sont alors pas sans rappeler le concept de gouvernance territoriale. Elles s'inscrivent, en effet, dans un cadre territorial précis et mettent en jeu des rapports de coopérations qui ne sont plus "ordonnés par la hiérarchie " (Pasquier, Simoulin \& Weisbein, 2007). En outre, elles rassemblent une multiplicité de partenaires dont "l'interaction est rendue nécessaire par le fait qu'aucun acteur [...] ne dispose [...] des connaissances et des moyens nécessaires pour s'attaquer seuls aux problèmes » (Leloup, Moyart \& Pecqueur, 2005).

En définitive, la posture de chacune des trois collectivités territoriales impliquées est significativement différente selon l'angle d'approche adopté. Au regard de la formation professionnelle, la Région semble légitime et s'affirme en tant que telle, alors que les Communautés d'agglomération et Départements pourraient, à première vue, paraittre en décalage. En revanche, si l'on se place du point de vue de la territorialisation, de la volonté du Conseil régional de nouer des partenariats, de créer des projets et de produire une politique de formation professionnelle en articulation avec les problématiques de développement économique et d'emploi, les Communautés et Départements sont en mesure de revendiquer une place importante. Celle-ci leur permet d'intervenir à différents niveaux selon les contextes particuliers à chaque territoire. 


\section{Conclusion}

Le territoire est un élément constitutif essentiel de l'action publique, que les acteurs mobilisent de plus en plus. Qu'il soit défini comme une entité géographique, administrative, comme un périmètre d'intervention, ou bien comme un lieu favorisant l'émergence de projet, il se retrouve presque partout dans les programmes politiques. Le suivi d'un dispositif dont l'objectif est la prise en compte de ce territoire offre à l'analyse un point de vue intéressant, celui des acteurs qui travaillent à la construction de ce dispositif et à celle de son territoire. Il permet de souligner l'importance des bricolages institutionnels qui sont à l'origine de cette fabrication d'un " territoire pertinent ", dont la définition sera inéluctablement amenée à évoluer en fonction des nombreuses dimensions que sont le contexte, les intérêts, ressources et stratégies des acteurs.

Ainsi, la construction du Programme de Formation Territorialisé (PFT) a pu faire voler en éclats le cadre protocolaire initialement institué par le Conseil régional : sur l'un des territoires d'observation, le PFT ne fait pas intervenir les acteurs de la Communauté d'agglomération, pourtant perçue comme une dimension constitutive du "territoire pertinent ", et s'étend sur une zone géographique qui ne correspond pas aux " 45 minutes de mobilité " antérieurement définies. En revanche, les acteurs et le « territoire " concernés reflètent la réalité d'un projet local, et les ressources mobilisables. En ce sens, le territoire correspond à "l'émanation d'une logique collective» (Bel, 2007) et non d'une démarche verticale.

Le processus de construction du dispositif révèle, en outre, la position des acteurs vis-à-vis du territoire et vient nuancer les propos de Friedberg (1988) relatifs au "modèle bureaucratique à la française ». Ce dernier estimait, en effet, que ce modèle se caractérisait, entre autres, par une grande inertie " car ceux qui perçoivent les transformations là où elles apparaissent, c'est-à-dire au niveau de l'exécution, n'ont pas le pouvoir d'introduire les changements nécessaires. Et ceux qui ont le pouvoir, c'est-à-dire les dirigeants du sommet, n'ont pas l'information pertinente, soit parce qu'on la leur cache, soit parce quils ne savent ni la recevoir ni l'utiliser». Or, si l'on considère, avec Bonoli et Palier (1999), que le changement peut être introduit par des transformations mineures, l'introduction du PFT pourrait bien venir constituer un changement important, significatif d'une capacité d'analyse croissante pour l'ensemble des acteurs concernés.

Des deux acceptions de la territorialisation - descendante et ascendante - chaque acteur - élu et technicien régionaux, acteurs locaux - ne semble retenir que celle qui correspond le plus à ses intérêts et qui peut constituer une ressource non négligeable. Ainsi, une dialectique de ces deux référentiels émerge. Elle contribue à l'élaboration d'une politique hybride qui emprunte autant aux deux logiques et demande à chaque partenaire de réévaluer sa position en fonction des spécificités de chacune des situations territoriales auxquelles il fait face. 


\section{Bibliographie}

Agulhon C., Trautman J. \& Vincent C. (2002), "Cahier régional XXX », dans le cadre de L'évaluation des politiques régionales de formation professionnelle 1999-2001 réalisé pour le Comité de coordination des programmes régionaux d'apprentissage et de formation professionnelle continue, Paris.

Bel M. (2007), « Formation et territoire : des approches renouvelées », Formation emploi, n'97, pp. 67-80.

Bel M., Dubouchet L. (dir.) (2004), Décentralisation de la formation professionnelle : un processus en voie d'achèvement? Aix-en-Provence, Editions de l'Aube.

Berthet T. (2010), « Focus - Territorialisation et changements dans l'action publique locale en matière sociale : l'exemple de la formation professionnelle ", Informations sociales, $\mathrm{n}^{\circ} 157$, pp. 90-92.

Catlla M. (2007), « De la genèse d'une régulation territorialisée à l'émergence d'une gouvernance territoriale ", in Pasquier R., Simoulin V. \& Weisbein J. (dir.) La gouvernance territoriale. Pratiques, Discours et Théories, Paris, LGDJ, coll. «Droit \& Société ».

Douillet A.-C. (2003), «Les élus ruraux face à la territorialisation de l'action publique ", Revue française de science politique, $\mathrm{n}^{\circ} 4$ vol. 53, pp. 583-606.

Friedberg E. (1988), «L'analyse sociologique des organisations », POUR, n² 28, pp. 3-125.

Hassenteufel P. (2008), Sociologie politique : l'action publique, Paris, Armand Colin, coll. «U $"$.

Jobert A. (2006), « Enjeux et dynamique du dialogue social territorial », POUR, $\mathrm{n}^{\circ} 192$, pp. 190-195.

Jobert A., Guarriello F. \& Heidling E. (2009), « Le dialogue social territorial en Europe : perspective comparative ", in Duclos L., Groux G. \& Mériaux O. (dir.) Les nouvelles dimensions du politique. Relations professionnelles et régulations sociales, Paris, LGDJ, coll. «Droit et Société ».

Lamanthe A. (2004), " Le fait régional français : entre découpages préexistants et territorialisation de l'action ", in Bel M., Dubouchet L. (dir.), Décentralisation de la formation professionnelle : un processus en voie d'achèvement? Aix-en-Provence, Editions de l'Aube.

Lascoumes P. \& Le Galès P. (dir.) (2004), Gouverner par les instruments, Sciences Po Paris.

Leloup F., Moyart L. \& Pecqueur B. (2005), « La gouvernance territoriale comme nouveau mode de coordination territoriale ?", Géographie Economie Société, n 4 vol. 7, pp. 321-331.

Mossé P., Verdier E. (2003), « Différenciations régionales ou permanences des spécificités sectorielles : formation des jeunes et politique hospitalière ", in Bel M., Méhaut P., 
Mériaux O. (coord.), La décentralisation de la formation professionnelle. Quels changements dans la conduite de l'action publique ?. L'Harmattan, coll. "Logiques politiques ", pp. $115-135$

Muller P. (2005), «Esquisse d'une théorie du changement dans l'action publique : structures, acteurs et cadres cognitifs ", Revue française de science politique, n ${ }^{\circ}$ 55, pp. 155-187.

Pasquier R., Simoulin V. \& Weisbein J. (dir.) (2007), La gouvernance territoriale. Pratiques, Discours et Théories, Paris, LGDJ, coll. " Droit \& Société ».

Palier B. \& Bonoli G. (1999), « Phénomènes de path dependence et réformes des systèmes de protection sociale ", Revue française de science politique, n³ 3, 1999, pp. 399-420.

Rey F. (2012), Définition de "Territoires ", in Bevort \& alii, Dictionnaire du travail, Paris, PUF,pp. 792-798.

Rose J. (2008), "La professionnalisation des études supérieures : tendances, acteurs, formes concrètes ", Relief, Céreq, n 25 , pp. 42-57. 